\title{
CARDIO-RESPIRATORY FITNESS IS ASSOCIATED WITH A VERBAL FACTOR ACROSS COGNITIVE DOMAINS IN SCHIZOPHRENIA
}

Tom Langerud Holmen ${ }^{1}$, MA, John Abel Engh ${ }^{1}$, MD PhD, Eivind Andersen ${ }^{3}, \mathrm{PhD}$, Ole Andreas Andreassen ${ }^{4}, \mathrm{MD} \mathrm{PhD}$, Egil Wilhelm Martinsen ${ }^{5}, \mathrm{MD} \mathrm{PhD}$, Therese Torgersen Bigseth $^{1}, \mathrm{MD}$, Gry Bang-Kittilsen ${ }^{1}, \mathrm{MA}$, and Jens Egeland ${ }^{1,2}, \mathrm{PhD}$

${ }^{1}$ Division of Mental Health and Addiction, Vestfold Hospital Trust, Tønsberg, Norway, ${ }^{2}$ Department of Psychology, University of Oslo, Oslo, Norway, ${ }^{3}$ Faculty of Humanities, Sports and Educational Science, University College of Southeast Norway, Norway, ${ }^{4}$ NORMENT, KG Jebsen Centre for Psychosis Research, Division of Mental Health and Addiction, Oslo University Hospital \& Institute of Clinical Medicine, University of Oslo, Oslo, Norway, ${ }^{5}$ Department of Research, Division of Mental Health and Addiction, Oslo University Hospital \& Institute of Clinical Medicine, University of Oslo, Oslo, Norway

Corresponding author:

Tom Langerud Holmen

Nordre Vestfold DPS

Postboks 164 Teie

3106 Nøtterøy

NORWAY

Telephone: (+47) 97119323

Fax: (+47) 33345152

Email: lantom@siv.no 
Objective: We investigated whether the relationship between cardio-respiratory fitness (CRF) and cognition in schizophrenia is general, or due to selective relationships between CRF and specific aspects of cognitive function.

Method: Eighty outpatients with schizophrenia spectrum disorders participated. Neurocognition was assessed with the Wechsler Adult Intelligence Scale version 4 General Ability Index, the MATRICS Consensus Cognitive Battery (MCCB) and the Emotion in Biological Motion (EBM) test. CRF was assessed with peak oxygen uptake measured directly during maximum exercise using a modified Balke protocol. Partial correlations, controlling for sex and age, were obtained for the perceptual and the verbal indices of WAIS GAI, six cognitive domains of $\mathrm{MCCB}$, and the EBM total score. A factor analysis was conducted on all 15 subtests of the WAIS GAI and the MCCB, and the factors were subjected to separate regression analyses with $\mathrm{CRF}$ as predictor.

Results: Significant, relatively similar and moderately sized correlations were found between CRF and social cognition, both indices of WAIS GAI, and five of the six cognitive domains of MCCB. The factor analysis identified three factors: one speed/attention/executive function factor, one verbal factor, and one perceptual factor. Regression analyses showed that $\mathrm{VO}_{2 \text { peak }}$ explained a significant amount of variance in the verbal factor only $\left(R^{2}=.06, \beta=.329\right.$, $p=.03)$.

Conclusion: The results indicate that the relationship between CRF and cognition in schizophrenia is selectively tied to a modality-specific association with verbal cognitive abilities. These findings have implications for understanding the relation between cognitive factors and physical health in schizophrenia.

Key words: psychosis/schizophrenia/cognition/exercise/CRF

ClinicalTrials.gov reg. number NCT02205684 (clinicaltrials.gov/ct2/show/NCT02205684) 


\section{Introduction}

At group level, individuals with schizophrenia display low cardio-respiratory fitness (CRF) (Andersen, 2018; Heggelund et al., 2011; Scheewe et al., 2012; Strassnig et al., 2011), and impaired cognition is a central clinical feature in schizophrenia (Aleman et al., 1999; Dickinson et al., 2007; Mesholam-Gately et al., 2009). Hence, the relationship between CRF and cognition is of particular interest with regard to this patient group. Intervention studies have reported associations between CRF and specific cognitive functions, both in the general population and in various patient groups (Hotting and Roder, 2013; Kramer et al., 1999; Malchow et al., 2015; Oertel-Knochel et al., 2014; Pajonk et al., 2010; Smiley-Oyen et al., 2008; Su et al., 2016; Voelcker-Rehage and Niemann, 2013). This has formed the basis for various models connecting CRF with cognition (Kramer et al., 1999; Voss, 2016). A metaanalysis on ten intervention studies with individuals with schizophrenia reported significant effects of increased CRF on overall cognitive performance, and for working memory, attention/vigilance and social cognition specifically (Firth et al., 2016).

However, we believe that the question of differential associations between CRF and specific cognitive functions has not yet been thoroughly investigated in this population. Depending on the particular study and the specific cognitive tests included, improvements in a large range of cognitive functions have been reported. Still, limited sample sizes and the varying selections of cognitive functions tested in the single studies constitute a suboptimal foundation for investigating differentiated associations between CRF and specific cognitive functions. Moreover, while intervention studies are necessary to evaluate the modifying effect of the variables in question, this approach may not be sufficient to illuminate the long-term, life-span developmental processes behind the same variables. The investigation of naturally occurring associations between $\mathrm{CRF}$ and cognitive functions may provide important 
information about the natural processes on which the intervention studies are designed to intervene. Addressing this issue in a cross-sectional study, Kimhy and colleagues investigated naturally occurring associations between CRF and the subset of cognitive functions included in the MATRICS Consensus Cognitive Battery (MCCB) in a sample of 32 schizophrenia patients, and reported positive partial correlations between CRF and the specific domains of executive functioning, working memory and processing speed, and also social cognition, as well as a trend level association to visual learning (Kimhy et al., 2014). The authors conveyed these findings as preliminary and called for replication in a larger sample. We wanted to examine this subject further by utilizing a more extensive test battery in a larger sample of individuals with schizophrenia, and a different methodological approach.

In a previous study, we found that CRF explained $8.2 \%$ and $9.0 \%$ of the variance in general intellectual ability (IQ) and state-sensitive cognitive functions (processing speed, attention, working memory, learning and executive functions) respectively (Holmen et al., 2017). The effect of CRF on cognition was significant beyond the impact of negative psychotic symptom load. Furthermore, years of education, duration of illness, body mass index (BMI) or cigarette smoking did not influence the relationship between CRF and cognition. The relationship between $\mathrm{CRF}$ and general intellectual ability was approximately as strong as the relationship between CRF and the more state-sensitive cognitive functions. This arguably indicates that the association is established developmentally early. However, other variations may exist within and across the different cognitive functions. Investigating such an effect may require a search beyond specific cognitive subtests, as no subtest is completely function-pure (Moscovitch, 1994). For example, a test measuring executive functions can include a visual-spatial component, and a test measuring perceptual abilities may include a processing speed component. An elucidating alternative may be to enter all subtests into a factor analysis and thereby search for underlying dimensions which cut across 
the divisions of single tests and domains. Identified factors may then be subjected to regression analyses, to assess and compare their respective relationships with CRF.

Firstly, we will examine the associations between CRF and each of the cognitive domains and thereby replicate the study of Kimhy and colleagues (Kimhy et al., 2014) in a larger sample, and extend it by also including associations to general intellectual ability. Secondly, in a search for potential underlying dimensions, we will subject all specific subtests to an exploratory factor analysis. Subsequently we will conduct regression analyses on all identified factors, with CRF as predictor.

\section{Method}

\subsection{Design}

The study was conducted using baseline data from the randomized, controlled, observer-blinded clinical trial 'Effects of Physical Activity in Psychosis' (EPHAPS) (ClinicalTrials.gov, registration number NCT02205684) (Engh et al., 2015).

\subsection{Participants}

Eighty participants aged 20-67 years were recruited from August 2014 through February 2017 from catchment area-based and publicly funded outpatient psychiatric clinics in Vestfold County, Norway. A subgroup of patients was referred from primary health care to the outpatient clinics for participation in the project specifically. Eligible for the study were patients who fulfilled the Diagnostic and Statistical Manual of Mental Disorders 5th ed. 
(DSM 5; American Psychiatric Association, 2013) criteria for schizophrenia spectrum disorder (schizophrenia, schizoaffective disorder, and schizophreniform disorder). Demographic and clinical characteristics are presented in Table 1. Diagnoses were established using the Structured Clinical Interview for DSM axis I disorders (SCID I) (First, 2002). Interviews were conducted by a clinical psychologist or a specialist in psychiatry. Members of the assessment staff attended the SCID training program from UCLA (Ventura et al., 1998), and all participated in diagnostic consensus meetings.

Additional inclusion criteria were: Age between 18 and 67; understanding and speaking a Scandinavian language. Exclusion criteria were: pregnancy; chest pain during CRF test; unstable angina pectoris; recent myocardial infarction; uncontrollable cardiac arrhythmia; severe hypertension (>180/110 mmHg); comorbid diagnosis of mild mental retardation; or other medical conditions incompatible with participation.

Seventy-seven participants received antipsychotic medical treatment. Defined daily doses (DDD) were calculated in accordance with guidelines from the World Health Organization Collaborating Center for Drug Statistics Methodology (http://www.whocc.no/atcdd). Additional regular anxiolytic and/or antidepressant or mood stabilizing medication was received by 9,15 and 11 participants, respectively. Eighteen participants received clozapine (12) or other types of medication (6) with potential anticholinergic effects.

The study was approved by the Regional Committee for Medical and Health Research Ethics of Southern and Eastern Norway (file number 2014/372/REK SØR-ØST). Initial information about the study was given to eligible patients by clinical staff in the outpatient 
clinic or in primary health services. Contingent on understanding the nature of the research and willingness to participate, written consent was obtained by a project coworker.

\section{Insert Table 1 about here.}

\subsection{Procedure}

The current study was based on neurocognitive and clinical assessments, and a CRF test, all conducted within a two-week period. Clinical symptoms were assessed with the Positive and Negative Syndrome Scale (PANSS) (Kay et al., 1987), and psychosocial functioning was assessed with the Global Assessment of Functioning scale (GAF) (Endicott et al., 1976). Neurocognition was assessed with the six subtests of the Wechsler Adult Intelligence Scale version 4 (2008, NCS Pearson Inc. San Antonio, TX, USA) included in the Verbal Comprehension and the Perceptual Reasoning sub-indices. Together, these comprise a General Ability Index that may be regarded as a core IQ measure not contaminated by statedependent reductions in processing speed and memory. Furthermore, nine subtests of the MATRICS Consensus Cognitive Battery were applied as measures of the state-sensitive cognitive functions, covering six domains: processing speed, attention/vigilance, working memory, verbal learning, visual learning and problem solving (Green et al., 2014). The Norwegian version of MCCB has retained the original psychometric properties (Holmen et al., 2010), with the possible exception of the test intended to measure social cognition, which was not included in the current study. Instead, an emotion-interpretation aspect of social cognition was measured utilizing the Emotion in Biological Motion (EBM) test. The test consists of 22 video clips of point-light display walkers, requiring scorers to interpret emotional tone by choosing from the five alternatives of 'angry', 'happy', 'sad', 'fearful' or 'neutral/no emotion' 
(Couture et al., 2010; Heberlein et al., 2004). Vaskinn and colleagues standardized the test on a Norwegian sample of 101 healthy controls, which was used as basis for calculating a total score for each participant (Vaskinn et al., 2015). A proportional scoring method was utilized; each of the participant's single item scores was rated on basis of the proportion of healthy controls that scored accordingly, and an individual total score was then calculated for each participant.

$\mathrm{CRF}$, as measured by peak oxygen uptake $\left(\mathrm{VO}_{2 \text { peak }}\right.$, calculated from the formula $\mathrm{ml} / \mathrm{kg} / \mathrm{min}$ ), was measured during a maximum exercise test on a treadmill using a modified Balke protocol (Balke and Ware, 1959), where speed was held constant at $5 \mathrm{~km} / \mathrm{h}$ and the inclination angle was increased by one degree every minute until exhaustion. Gas exchange was sampled continuously into a mixing chamber every $30 \mathrm{~s}$ by having the participants breathe into a Hans Rudolph 2-way breathing valve (2700 series, Hans Rudolph Inc., Kansas City, MO, USA) connected to a Jaeger Oxycon Pro gas analyzer (Erich Jaeger GmbH, Hoechberg, Germany), which measures the oxygen and carbon dioxide content. Before conducting the CRF test, somatic health was thoroughly assessed through general medical examination, including medical history, medication record, and physical examination with blood pressure assessment and electrocardiography. For various reasons some individuals may fail to reach a true $\mathrm{VO}_{2 \max }$, and for the sake of conservative reporting we therefore use the term $\mathrm{VO}_{2 \text { peak }}$.

For one participant, the data from the MCCB visual learning subtest BVMT-R was missing. To retain the individual's data in all analyses the entry was substituted with a calculated mean of t-scores of the remaining eight MCCB subtests. 


\subsection{Statistical Analysis}

Analyses were made using The Statistical Package for the Social Sciences (IBM SPSS Version 22.0, IBM Corp, Armonk, NY, USA). Visual inspection of histograms and values of skewness and kurtosis indicated that all data were normally distributed, and no outliers needed to be addressed. For purpose of a general overview, partial correlations, controlling for sex and age, were obtained for the Verbal Comprehension (VCI) and the Perceptual Reasoning (PRI) indices of the WAIS IV, the six domain scores of the Neurocognitive Composite Score of MCCB (MCCB NCS), and the EBM total score, respectively.

Significance tests were set as one-tailed, as predicted direction of the associations was given from the results of a previous study (Holmen et al., 2017). In this previous study we also controlled for a range of possible confounders, which were shown not to exert significant influence on the general relationship between CRF and cognition. We therefore did not control for these variables in the current context. Next, the six subtests of the WAIS VCI and PRI and the nine MCCB NCS subtests were deemed suitable for factoring according to the Kaiser-Meyer-Olkin measure $(\mathrm{KMO}=.91)$ and subjected to a principal axis factoring analysis using Varimax rotation. The main purpose of the factor analysis was to search for underlying cognitive factors extending across the single domains and tests. Inherently, it is also a correction for multiple testing, reducing the number of variables to a statistically manageable number for further analyses. Factors with Eigenvalues $>1$ were retained and subsequently subjected to regression analyses, with each factor as dependent variable and $\mathrm{VO}_{2 \text { peak }}$ as predictor.

\section{Results}


Partial correlations are displayed in Table 2, showing significant correlations between $\mathrm{VO}_{2 \text { peak }}$ and both the VCI and PRI indexes of the WAIS and five of the six MCCB NCS domains. All significant correlations are positive and fairly similar in size, ranging from $r$ $=.23$ to $r=.29$. The domain of processing speed is close to reaching significance $(r=.17, p$ $=.06)$. The social cognition measure of the EBM total score also emerges with a significant positive correlation to $\mathrm{VO}_{2 \text { peak }}(r=.21, p=0.04)$.

The factor analysis of all 15 subtests reveals three factors: one whereon processing speed, attention, working memory and executive function loaded highly (Eigenvalue $=7.0$, $46.7 \%$ of variance explained); one appearing as a verbal factor (Eigenvalue $=1.3,8.6 \%$ of variance explained); and one appearing as a perceptual factor (Eigenvalue $=1.2,7.9 \%$ of variance explained). Factor loadings are displayed in Table 3. Controlling for sex and age, regression analyses showed that $\mathrm{VO}_{2 \text { peak }}$ significantly predicted $6 \%$ of the variance of the verbal factor $\left(R^{2}=.060, \beta=.329, F\right.$-change $\left.(3,76)=5.110, p=.03\right)$. The other two factors were not significantly related to $\mathrm{VO}_{2 \text { peak }}$ in this material (speed/attention/executive function factor: $R^{2}=.028, \beta=.221, F$-change $(3,76)=2.132, p=.15$; perceptual factor: $R^{2}=.013, \beta=.154, F$-change $\left.(3,76)=1.173, p=.28\right)$.

Insert Tables 2 and 3 about here.

\section{Discussion}

$\mathrm{CRF}$, as measured by $\mathrm{VO}_{2 \text { peak, }}$ was significantly associated with the whole span of cognitive domains, with the exception of the domain of processing speed, where a trend-level association appeared in the expected direction. These results indicate that the association 
between CRF and cognition is evenly distributed across the cognitive indices and domains. Also the EBM test of emotion interpretation, which constitutes one aspect of social cognition, showed a significant but small relationship to CRF. Thus, the main trend in the preliminary results from the study of Kimhy and colleagues (Kimhy et al., 2014) was replicated in our larger sample, and shown to extend also to general intellectual ability/IQ. Despite not reaching significance for the domain of processing speed, also this result was considered to be consistent with the overall trend of generally distributed associations.

By splitting up the cognitive indices and domains into the separate subtests, subjecting these to a factor analysis and conducting regression analyses on the factor scores, we were able to investigate the associations in further detail. We thereby discovered a modalityspecific association not visible in the bivariate analyses. The overall association between CRF and cognition appeared selectively attributed to a modality-specific relationship between CRF and a verbal cognitive factor. This underlying factor extends across the division between general intellectual ability and state-sensitive cognitive functions, and also across singular cognitive domains and functions.

This result corresponds with the finding that the Verbal Comprehension Index of WAIS IV had the largest single partial correlation to CRF. We do not know why the association between CRF and cognition appears selectively tied to a verbal factor. It is contrary to a proximal modality effect of CRF on cognitive abilities more activated during exercise, as suggested by some theories in the field; the selective improvements hypothesis (Kramer et al., 1999) and the motor fitness hypothesis (Voss, 2016) hold that various exercise activities may induce selective improvements in executive functions and/or perceptual cognitive abilities. Conversely, from the current results, the association appears selectively linked to the verbal modality. Interestingly, Pajonk and colleagues found significant increases 
in hippocampal volume both in people with schizophrenia and healthy participants following an exercise intervention, as compared to no changes in a control group with individuals with schizophrenia patients playing table soccer (Pajonk et al., 2010). The exercise condition was followed by significantly improved performance on a verbal short-term memory test for the individuals with schizophrenia only (possibly due to a ceiling effect of the particular test for the healthy controls). Moreover, improvements in verbal short-term memory were significantly associated with the increases in hippocampal volume in the individuals with schizophrenia. More generally, in a recent meta-analysis on the effect of exercise on hippocampal volume in the general population, Firth and colleagues found a significant positive effect on left hippocampal volume exclusively (Firth et al., 2018). Primary language centers are located in the dominant left hemisphere in most of the human population, and there are indications that the left side of hippocampus is particularly important to verbal learning and verbal memory (Ezzati et al., 2016; Rausch and Babb, 1993; Saling et al., 1993; Sass et al., 1992). Thus, previous lines of research have provided clues to a link between CRF and verbal cognitive abilities both in individuals with schizophrenia and in the general population. Furthermore, at group level, verbal memory is among the cognitive functions most severely affected in schizophrenia (Dickinson, 2013; Kravariti et al., 2009). This may fortify any specific relation between verbal cognitive abilities and CRF existing in the general population; individuals with schizophrenia may have more to gain in language-related cognitive functioning by improving their CRF.

In a previous study, we found that $\mathrm{CRF}$ explained as much variance in general intellectual ability as in the state-sensitive cognitive functions (Holmen et al., 2017). We interpreted this finding as an indication that the association between CRF and cognition in schizophrenia is established at the time when general intellectual ability is consolidated in the young person. This implies that genes in interaction with earlier environmental circumstances 
may be more influential on this relationship, than recent or current life-style. Moreover, heritability estimates of the perceptual and verbal components of general intellectual ability are relatively similar in size (Plomin, 2000), while the results of the present study indicate that only the latter is significantly related to CRF. Åberg and colleagues controlled for genetic heritability in their large-scale cohort study, and concluded that the correlations between CRF and cognition in the general population are predominantly determined by the non-shared environment (Aberg et al., 2009). Taken together, this arguably points to a possible primacy of environmental and life-style factors, particularly during childhood, adolescence and early adulthood, in establishing the relationship between CRF and cognition in schizophrenia.

The study has several limitations. In the absence of a control group and other relevant studies on the particular topic, we do not know whether the selective association between CRF and verbal cognitive abilities are specific for individuals with schizophrenia or also applies to the general population. The cross-sectional design of the study leaves any propositions about causal pathways inferential. Several possible third-variable influences on the relationship between CRF and cognition in schizophrenia have been controlled for in a previous study (Holmen et al., 2017), but the possibility of other disorder-specific third variables contributing to the association, such as genetic factors, cannot be ruled out. Furthermore, in the present study we controlled for age and sex in all analyses, to account for general sex- and age-differences in $\mathrm{CRF}$ as measured by $\mathrm{VO}_{2 \text { peak. }}$. All cognitive measures used in the analyses are normed, thus they are age corrected. If there is a direct relationship between $\mathrm{VO}_{2 \text { peak }}$ and aspects of cognition decaying with age, this effect may have been controlled away in the analyses. Changes in processing speed, which was the only cognitive function not associated with $\mathrm{VO}_{2 \text { peak }}$ in this study, is often considered the underlying mechanism mediating all cognitive changes in aging (Salthouse, 2008). It is possible that some of this effect is directly and causally related to an age-contingent reduction in oxygen 
uptake, as measured by $\mathrm{VO}_{2 \text { peak. }}$ Such an effect would have been controlled away when controlling for age. Hence, using a normed speed measure corrected for the large changes between young and old age, and correcting for age when considering the association to $\mathrm{VO}_{2 \text { peak }}$, may have deflated genuine associations that would be present in a more age homogeneous sample.

Conclusively, the broad association between CRF and several cognitive domains in schizophrenia seems to be driven by a verbal cognitive factor which was specifically identified in the factor analysis. Possible underlying mechanisms should be investigated in experimental studies.

Aberg, M.A., Pedersen, N.L., Toren, K., Svartengren, M., Backstrand, B., Johnsson, T., Cooper-Kuhn, C.M., Aberg, N.D., Nilsson, M., Kuhn, H.G., 2009. Cardiovascular fitness is associated with cognition in young adulthood. Proceedings of the National Academy of Sciences of the United States of America 106(49), 20906-20911.

Aleman, A., Hijman, R., de Haan, E.H., Kahn, R.S., 1999. Memory impairment in schizophrenia: a meta-analysis. The American journal of psychiatry 156(9), 1358-1366.

Andersen, E., Holmen, T. L., Egeland, J., Martinsen, E. W., Bigseth, T. T., Bang-Kittilsen, G., Anderssen, S. A., Hansen, B. A., Engh, J. A., 2018. Physical activity pattern and cardiorespiratory fitness in individuals with schizophrenia compared with a population-based sample. Under consideration for publication.

Balke, B., Ware, R.W., 1959. An experimental study of physical fitness of Air Force personnel. United States Armed Forces medical journal 10(6), 675-688.

Couture, S.M., Penn, D.L., Losh, M., Adolphs, R., Hurley, R., Piven, J., 2010. Comparison of social cognitive functioning in schizophrenia and high functioning autism: more convergence than divergence. Psychological medicine 40(4), 569-579.

Dickinson, D., Ramsey, M.E., Gold, J.M., 2007. Overlooking the obvious: a meta-analytic comparison of digit symbol coding tasks and other cognitive measures in schizophrenia. Archives of general psychiatry 64(5), 532-542.

Dickinson, D., Schaefer, J., Weinberger, D. R., 2013. The multi-faceted, "global" cognitive impairment in schizophrenia. In: Cognitive Impairment in Schizophrenia : Characteristics, Assessment and Treatment. Cambridge University Press, Cambridge. 
Endicott, J., Spitzer, R.L., Fleiss, J.L., Cohen, J., 1976. The global assessment scale. A procedure for measuring overall severity of psychiatric disturbance. Archives of general psychiatry 33(6), 766-771. Engh, J.A., Andersen, E., Holmen, T.L., Martinsen, E.W., Mordal, J., Morken, G., Egeland, J., 2015. Effects of high-intensity aerobic exercise on psychotic symptoms and neurocognition in outpatients with schizophrenia: study protocol for a randomized controlled trial. Trials 16, 557.

Ezzati, A., Katz, M.J., Zammit, A.R., Lipton, M.L., Zimmerman, M.E., Sliwinski, M.J., Lipton, R.B., 2016. Differential association of left and right hippocampal volumes with verbal episodic and spatial memory in older adults. Neuropsychologia 93(Pt B), 380-385.

First, M.B., Spitzer, R. L., Gibbon, M., Williams, J. B. W., 2002. Structured Clinical Interview for DSMIV-TR Axis 1 Disorders, Research Version, Patient Edition (SCID I/P). Biometrics Research, New York State Psychiatric Institute, New York.

Firth, J., Stubbs, B., Rosenbaum, S., Vancampfort, D., Malchow, B., Schuch, F., Elliott, R., Nuechterlein, K.H., Yung, A.R., 2016. Aerobic Exercise Improves Cognitive Functioning in People With Schizophrenia: A Systematic Review and Meta-Analysis. Schizophrenia bulletin.

Firth, J., Stubbs, B., Vancampfort, D., Schuch, F., Lagopoulos, J., Rosenbaum, S., Ward, P.B., 2018. Effect of aerobic exercise on hippocampal volume in humans: A systematic review and meta-analysis. Neurolmage 166, 230-238.

Green, M.F., Harris, J.G., Nuechterlein, K.H., 2014. The MATRICS consensus cognitive battery: what we know 6 years later. The American journal of psychiatry 171(11), 1151-1154.

Heberlein, A.S., Adolphs, R., Tranel, D., Damasio, H., 2004. Cortical regions for judgments of emotions and personality traits from point-light walkers. Journal of cognitive neuroscience 16(7), 1143-1158. Heggelund, J., Hoff, J., Helgerud, J., Nilsberg, G.E., Morken, G., 2011. Reduced peak oxygen uptake and implications for cardiovascular health and quality of life in patients with schizophrenia. BMC psychiatry 11, 188.

Holmen, A., Juuhl-Langseth, M., Thormodsen, R., Melle, I., Rund, B.R., 2010. Neuropsychological profile in early-onset schizophrenia-spectrum disorders: measured with the MATRICS battery. Schizophrenia bulletin 36(4), 852-859.

Holmen, T.L., Egeland, J., Andersen, E., Bigseth, T.T., Engh, J.A., 2017. The association between cardio-respiratory fitness and cognition in schizophrenia. Schizophrenia research.

Hotting, K., Roder, B., 2013. Beneficial effects of physical exercise on neuroplasticity and cognition. Neuroscience and biobehavioral reviews 37(9 Pt B), 2243-2257.

Kay, S.R., Fiszbein, A., Opler, L.A., 1987. The positive and negative syndrome scale (PANSS) for schizophrenia. Schizophrenia bulletin 13(2), 261-276.

Kimhy, D., Vakhrusheva, J., Bartels, M.N., Armstrong, H.F., Ballon, J.S., Khan, S., Chang, R.W., Hansen, M.C., Ayanruoh, L., Smith, E.E., Sloan, R.P., 2014. Aerobic fitness and body mass index in individuals with schizophrenia: Implications for neurocognition and daily functioning. Psychiatry research 220(3), 784-791.

Kramer, A.F., Hahn, S., Cohen, N.J., Banich, M.T., McAuley, E., Harrison, C.R., Chason, J., Vakil, E., Bardell, L., Boileau, R.A., Colcombe, A., 1999. Ageing, fitness and neurocognitive function. Nature 400(6743), 418-419.

Kravariti, E., Morgan, K., Fearon, P., Zanelli, J.W., Lappin, J.M., Dazzan, P., Morgan, C., Doody, G.A., Harrison, G., Jones, P.B., Murray, R.M., Reichenberg, A., 2009. Neuropsychological functioning in first-episode schizophrenia. The British journal of psychiatry : the journal of mental science 195(4), 336-345.

Malchow, B., Keller, K., Hasan, A., Dorfler, S., Schneider-Axmann, T., Hillmer-Vogel, U., Honer, W.G., Schulze, T.G., Niklas, A., Wobrock, T., Schmitt, A., Falkai, P., 2015. Effects of Endurance Training Combined With Cognitive Remediation on Everyday Functioning, Symptoms, and Cognition in Multiepisode Schizophrenia Patients. Schizophrenia bulletin 41(4), 847-858.

Mesholam-Gately, R.I., Giuliano, A.J., Goff, K.P., Faraone, S.V., Seidman, L.J., 2009. Neurocognition in first-episode schizophrenia: a meta-analytic review. Neuropsychology 23(3), 315-336. 
Moscovitch, M., 1994. Memory and working with memory: Evaluation of a component process model and comparison with other models. In: Memory systems 1994. MIT Press, Cambridge, Mass. Oertel-Knochel, V., Mehler, P., Thiel, C., Steinbrecher, K., Malchow, B., Tesky, V., Ademmer, K., Prvulovic, D., Banzer, W., Zopf, Y., Schmitt, A., Hansel, F., 2014. Effects of aerobic exercise on cognitive performance and individual psychopathology in depressive and schizophrenia patients. European archives of psychiatry and clinical neuroscience 264(7), 589-604.

Pajonk, F.G., Wobrock, T., Gruber, O., Scherk, H., Berner, D., Kaizl, I., Kierer, A., Muller, S., Oest, M., Meyer, T., Backens, M., Schneider-Axmann, T., Thornton, A.E., Honer, W.G., Falkai, P., 2010.

Hippocampal plasticity in response to exercise in schizophrenia. Archives of general psychiatry 67(2), 133-143.

Plomin, R., DeFries, J. C., McClearn, G. E., McGuffin, P., 2000. Behavioral genetics, Fourth edition. ed. Worth Publishers, New York, NY.

Rausch, R., Babb, T.L., 1993. Hippocampal neuron loss and memory scores before and after temporal lobe surgery for epilepsy. Archives of neurology 50(8), 812-817.

Saling, M.M., Berkovic, S.F., O'Shea, M.F., Kalnins, R.M., Darby, D.G., Bladin, P.F., 1993. Lateralization of verbal memory and unilateral hippocampal sclerosis: evidence of task-specific effects. Journal of clinical and experimental neuropsychology 15(4), 608-618.

Salthouse, T.A., Madden, D. J., 2008. Information processing speed and aging. In: Information Processing Speed in Clinical Populations (Studies on Neuropsychology, Neurology and Cognition). US: Taylor \& Francis, Philadelphia, PA.

Sass, K.J., Sass, A., Westerveld, M., Lencz, T., Novelly, R.A., Kim, J.H., Spencer, D.D., 1992. Specificity in the correlation of verbal memory and hippocampal neuron loss: dissociation of memory, language, and verbal intellectual ability. Journal of clinical and experimental neuropsychology 14(5), 662-672.

Scheewe, T.W., Takken, T., Kahn, R.S., Cahn, W., Backx, F.J., 2012. Effects of exercise therapy on cardiorespiratory fitness in patients with schizophrenia. Medicine and science in sports and exercise 44(10), 1834-1842.

Smiley-Oyen, A.L., Lowry, K.A., Francois, S.J., Kohut, M.L., Ekkekakis, P., 2008. Exercise, fitness, and neurocognitive function in older adults: the "selective improvement" and "cardiovascular fitness" hypotheses. Annals of behavioral medicine : a publication of the Society of Behavioral Medicine 36(3), 280-291.

Strassnig, M., Brar, J.S., Ganguli, R., 2011. Low cardiorespiratory fitness and physical functional capacity in obese patients with schizophrenia. Schizophrenia research 126(1-3), 103-109.

Su, C.Y., Wang, P.W., Lin, Y.J., Tang, T.C., Liu, M.F., Chen, M.D., 2016. The effects of aerobic exercise on cognition in schizophrenia: A 3-month follow-up study. Psychiatry research 244, 394-402.

Vaskinn, A., Sundet, K., Ostefjells, T., Nymo, K., Melle, I., Ueland, T., 2015. Reading Emotions from Body Movement: A Generalized Impairment in Schizophrenia. Frontiers in psychology 6, 2058. Ventura, J., Liberman, R.P., Green, M.F., Shaner, A., Mintz, J., 1998. Training and quality assurance with the Structured Clinical Interview for DSM-IV (SCID-I/P). Psychiatry research 79(2), 163-173. Voelcker-Rehage, C., Niemann, C., 2013. Structural and functional brain changes related to different types of physical activity across the life span. Neuroscience and biobehavioral reviews $37(9 \mathrm{Pt} \mathrm{B})$, 2268-2295.

Voss, M.W., 2016. The Chronic Exercise-Cognition Interaction: fMRI research. In: Exercise-cognition interaction : neuroscience perspectives. Elsevier, Academic Press, London, UK. 